\title{
Pre-eclampsia and risk of later kidney disease: nationwide cohort study
}

Check for updates

${ }^{1}$ Department of Epidemiology Research, Statens Serum Institut, Artillerivej 5, DK-2300 Copenhagen S, Denmark

${ }^{2}$ Department of Nephrology, Copenhagen University Hospital (Rigshospitalet), Blemdamsvej 9, DK-2100 Copenhagen $\varnothing$, Denmark Correspondence to: $\mathrm{H}$ Boyd hoy@ssi.dk

(ORCID 0000-0001-6849-9985) Additional material is published online only. To view please visit the journal online.

Cite this as: BMJ 2019;365:11516 http://dx.doi.org/10.1136/bmj.l1516

Accepted: 19 March 2019
Jonas H Kristensen, ${ }^{1}$ Saima Basit, ${ }^{1}$ Jan Wohlfahrt, ${ }^{1}$ Mette Brimnes Damholt, ${ }^{2}$ Heather A Boyd ${ }^{1}$

\section{ABSTRACT}

OBJECTIVE

To investigate associations between pre-eclampsia and later risk of kidney disease.

DESIGN

Nationwide register based cohort study.

SETTING

Denmark.

\section{POPULATION}

All women with at least one pregnancy lasting at least 20 weeks between 1978 and 2015 .

MAIN OUTCOME MEASURE

Hazard ratios comparing rates of kidney disease between women with and without a history of preeclampsia, stratified by gestational age at delivery and estimated using Cox regression.

RESULTS

The cohort consisted of 1072330 women followed for 19994470 person years (average 18.6 years/ woman). Compared with women with no previous pre-eclampsia, those with a history of pre-eclampsia were more likely to develop chronic renal conditions: hazard ratio 3.93 (95\% confidence interval 2.90 to 5.33 , for early preterm pre-eclampsia (delivery $<34$ weeks); 2.81 (2.13 to 3.71) for late preterm preeclampsia (delivery 34-36 weeks); 2.27 (2.02 to 2.55 ) for term pre-eclampsia (delivery $\geq 37$ weeks). In particular, strong associations were observed for chronic kidney disease, hypertensive kidney disease, and glomerular/proteinuric disease. Adjustment for cardiovascular disease and hypertension only partially attenuated the observed associations. Stratifying the analyses on time since pregnancy showed that

\section{WHAT IS ALREADY KNOWN ON THIS TOPIC}

Epidemiological studies have documented strong associations between preeclampsia and end stage renal disease

Studies investigating associations between pre-eclampsia and both chronic kidney disease and clinical markers of renal dysfunction have produced highly variable results

This is probably due to considerable between study differences in sample size, length of follow-up, time since affected pregnancy, and endpoint definition

\section{WHAT THIS STUDY ADDS}

History of pre-eclampsia (particularly early preterm pre-eclampsia) was associated with greatly increased risks of chronic kidney disease, hypertensive kidney disease, and glomerular and proteinuric disease later in life By contrast, pre-eclampsia was only modestly associated with acute kidney disease

Associations between pre-eclampsia and chronic kidney disease and glomerular and proteinuric disease were especially strong within five years of the latest pregnancy associations between pre-eclampsia and chronic kidney disease and glomerular/proteinuric disease were much stronger within five years of the latest pregnancy (hazard ratio 6.11 (3.84 to 9.72) and 4.77 (3.88 to 5.86), respectively) than five years or longer after the latest pregnancy (2.06 (1.69 to 2.50) and 1.50 (1.19 to 1.88). By contrast, associations between pre-eclampsia and acute renal conditions were modest.

\section{CONCLUSION}

s Pre-eclampsia, particularly early preterm preeclampsia, was strongly associated with several chronic renal disorders later in life. More research is needed to determine which women are most likely to develop kidney disease after pre-eclampsia, what mechanisms underlie the association, and what clinical follow-up and interventions (and in what timeframe post-pregnancy) would be most appropriate and effective.

\section{Introduction}

Pre-eclampsia is a systemic disorder of pregnancy characterised by various manifestations of organ dysfunction, chief among them hypertension and proteinuria, a sign of renal dysfunction. ${ }^{1}$ Although a certain degree of glomerular endotheliosis may not be uncommon even in healthy pregnant women, ${ }^{2}$ women with pre-eclampsia show signs of severe endothelial dysfunction in the glomerulus, changes in expression of podocyte associated proteins, and possibly even podocyte injury and loss. ${ }^{2-5}$ In addition to significant proteinuria, women with pre-eclampsia can develop impaired glomerular filtration and, in severe cases, renal failure.

Although the renal dysfunction associated with pre-eclampsia is well documented, the repercussions of pre-eclampsia for later renal function and disease remain unclear. The potential renal consequences of pre-eclampsia include acute renal dysfunction that develops during the affected pregnancy and does not resolve after delivery, a lowering of the threshold for acute renal dysfunction given renal insult in the years after delivery, and increased susceptibility to later chronic kidney disease. Owing to large between study variation in sample size, length of follow-up, time since affected pregnancy, and outcome, studies of estimated glomerular filtration rate and albumin-creatinine ratio measured postpartum have yielded mixed results. When estimated glomerular filtration rate and albumincreatinine ratio were measured an average of 10 months postpartum, $13.7 \%$ of women with previous pre-eclampsia met KDIGO (Kidney Disease: Improving Global Outcomes) criteria for starting annual monitoring of renal function (albumin-creatinine ratio between 
30 and $300 \mathrm{mg} / \mathrm{g}$ or estimated glomerular filtration rate between 45 and $59 \mathrm{~mL} / \mathrm{min} / 1.73 \mathrm{~m}^{2}$; higher albumin-creatinine ratio or lower estimated glomerular filtration rate triggers even more frequent monitoring or specialist referral). ${ }^{67}$ By contrast, other studies found little evidence of diminished glomerular filtration or microalbuminuria in women with previous preeclampsia four and 10 years postpartum. ${ }^{89}$ However, a meta-analysis suggested that although serum creatinine and estimated glomerular filtration rate measured an average of seven years postpartum might not differ for women with and without a history of pre-eclampsia, women with previous pre-eclampsia had four times the risk of postpartum microalbuminuria. ${ }^{10}$

Studies of hypertensive disorders of pregnancy (which include gestational hypertension, preeclampsia, eclampsia, and the HELLP (haemolysis, elevated liver enzymes, and low platelets) syndrome) and end stage renal disease have documented strong associations, particularly for pre-eclampsia. ${ }^{11-13}$ By contrast, results from studies of chronic kidney disease have varied considerably, depending on sample size, endpoint definition, length of follow-up, and time since the affected pregnancy. Thus, some studies found no association between previous hypertensive disorders of pregnancy and later chronic kidney disease, ${ }^{14} 15$ whereas others reported strong associations, with up to ninefold increases in risk of chronic kidney disease. ${ }^{16}$

Pregnancy is often considered a cardiovascular stress test, the failure of which (for example, due to development of pre-eclampsia or gestational diabetes) has implications for a woman's future risk of cardiovascular disease. Mechanistic implications aside, evidence of strong associations between preeclampsia and kidney disease would suggest that knowledge of a woman's pregnancy experiences might also help clinicians to identify a group of women at risk of future kidney disease. We did a nationwide register based cohort study to explore the association between pre-eclampsia and later kidney disease, by gestational age at delivery (an indicator of severity of pre-eclampsia), type of kidney disease, and time since pregnancy.

\section{Methods}

\section{Study cohort}

Using the Medical Birth Register and the National Patient Register, ${ }^{17}{ }^{18}$ we identified all women with at least one pregnancy lasting at least 20 weeks and ending in live birth or stillbirth in Denmark during the period January 1978 to October 2015. Women registered in the National Patient Register with kidney disease before the start of follow-up (see Statistical analyses) were excluded from the cohort (fig 1). We also excluded women who were ever registered in the National Patient Register with congenital or hereditary conditions associated with kidney defects or insufficiency and women registered with cardiovascular disease, hypertension, autoimmune disease, or other conditions known or suspected to be related to both kidney disease and hypertensive disorders of pregnancy, before the start of follow-up (fig 1). (See supplementary methods for information on the registers used and definitions of exclusion conditions.)

\section{Pre-eclampsia (exposure)}

We identified women who had pre-eclampsia, eclampsia, or the HELLP syndrome during the study period by using the National Patient Register; we grouped eclampsia and the HELLP syndrome with pre-eclampsia because these conditions are rare in Denmark. Pre-eclampsia diagnoses in the National Patient Register have previously been validated against the American Congress of Obstetricians and Gynecologists' 2002 definitions, with good results. ${ }^{19}$ To ensure that registered diagnoses reflected true instances of pre-eclampsia, we required women to have at least one diagnosis registered in the period from one month before delivery to seven days postpartum. We did not consider women with diagnoses registered earlier in pregnancy without a confirmatory diagnosis registered in this period to have had pre-eclampsia. As, by definition, a diagnosis of pre-eclampsia is not assigned before 20 weeks' gestation, we considered only diagnoses registered after this point. (See supplementary methods for the relevant international classification of disease (ICD) codes.) We treated history of pre-eclampsia as a time dependent variable, such that a woman could contribute pregnancies and person time to both the unexposed and exposed groups during the follow-up period. However, once she had experienced pre-eclampsia, she contributed all remaining person time to the exposed group, regardless of any later normotensive pregnancies.

\section{Kidney disease (outcome)}

We considered a woman to have incident postpregnancy kidney disease if she received a first diagnosis of kidney disease in the National Patient Register or the Causes of Death Register ${ }^{20}$ more than three months after delivery. Diagnoses of moderate and severe renal disease in the National Patient Register, including hypertensive kidney disease, most glomerular disease diagnoses, chronic tubulointerstitial nephritis, and renal failure, have previously been validated. ${ }^{21}$ Although the study was small (50 records validated), diagnoses from $100 \%$ of the charts had been correctly coded and registered.

Because pre-eclampsia often involves kidney dysfunction, which in a proportion of women is severe and may even warrant a diagnosis of acute kidney failure, we could not disentangle incident kidney disease diagnoses assigned during pregnancy or shortly after delivery from diagnoses recorded to describe morbidity associated with pre-eclampsia. Consequently, our study focused only on diagnoses registered more than three months after delivery, under the assumption that any transient kidney dysfunction related to pre-eclampsia should have subsided by this point, any persistent problems would be registered again when postpartum kidney 
function did not normalise, and diagnoses registered for the first time more than three months after delivery were unlikely to describe morbidity related to preeclampsia. We considered all kidney disease registered in the peripartum period ( 20 weeks before delivery to three months postpartum) to be potentially pregnancy related and not of interest. Women registered with kidney disease within three months of their first delivery in the study period (that is, before the start of follow-up) were excluded from the study cohort; if a woman was registered with kidney disease in the peripartum period of any subsequent pregnancy, her follow-up ended.

In our cohort, $85 \%$ of women registered as having kidney disease had either a functional diagnosis or an aetiological diagnosis, but not both. (This is probably because establishing an aetiological diagnosis often requires a biopsy, which is not always feasible, resulting in assignment of a functional diagnosis instead. Conversely, when an aetiological diagnosis can be established, a functional diagnosis becomes redundant.) Consequently, our outcome categories included four functional categories (acute kidney failure, chronic kidney disease, hypertensive kidney disease, and unspecified kidney disease) and five aetiological categories (glomerular and proteinuric disease, acute tubulointerstitial nephritis, chronic tubulointerstitial nephritis, unspecified tubulointerstitial nephritis, and other kidney disease), as well as a separate group containing kidney disease due to external causes or secondary to known disease (for example, obstructive uropathy, nephropathy due to external toxicity, diabetic kidney disease). (See supplementary methods for the ICD codes used to define each category.) Women with both a functional diagnosis and an aetiological diagnosis were classified on the basis of the first registered diagnosis.

In our analyses, before considering the individual categories, we examined two broad outcome categories: acute renal disorders, which included acute kidney failure and acute tubulointerstitial nephritis; and chronic renal disorders, which encompassed chronic kidney disease, hypertensive kidney disease, chronic tubulointerstitial nephritis, and glomerular and proteinuric diseases.

\section{Statistical analyses}

We followed women from three months after their first delivery in the study period until the first of diagnosis of kidney disease, death, emigration, designated "missing" in the Civil Registration System, ${ }^{22}$ pregnancy with unknown gestational length, or 31 December 2015. Women with more than one pregnancy during the follow-up period contributed follow-up time during and after all pregnancies subsequent to the first, but the peripartum time associated with any subsequent pregnancies ( 20 weeks before delivery to three months after delivery) was excluded from the analyses. If a woman developed kidney disease in the peripartum period, follow-up ended.

Using Cox regression, we first estimated hazard ratios for acute renal disorders in aggregate and chronic renal disorders in aggregate, comparing women with and without a history of pre-eclampsia. We then estimated separate hazard ratios for individual kidney disease categories by using a competing risks approach-that is, in the analysis of each specific category, follow-up stopped when a woman received her first diagnosis of kidney disease regardless of type of kidney disease, but only a diagnosis in the specific disease category being analysed counted as a case.

In all models, we used age as the underlying time and stratified the baseline hazards by parity (one, two, or three or more live births and/or stillbirths; time dependent variable) and maternal birth year (five year intervals; time independent variable). This ensured that we compared rates in women of the same age, birth cohort, and parity; including maternal birth year also helped to account for possible time trends in pre-eclampsia and kidney disease diagnoses. All analyses were also adjusted for history of gestational hypertension and history of shortest gestation $(<29$ weeks, 30-31 weeks, 32-33 weeks, one week intervals from 34 weeks to 41 weeks, $\geq 42$ weeks; time dependent variable). We considered a woman to have gestational hypertension in a given pregnancy if she was registered

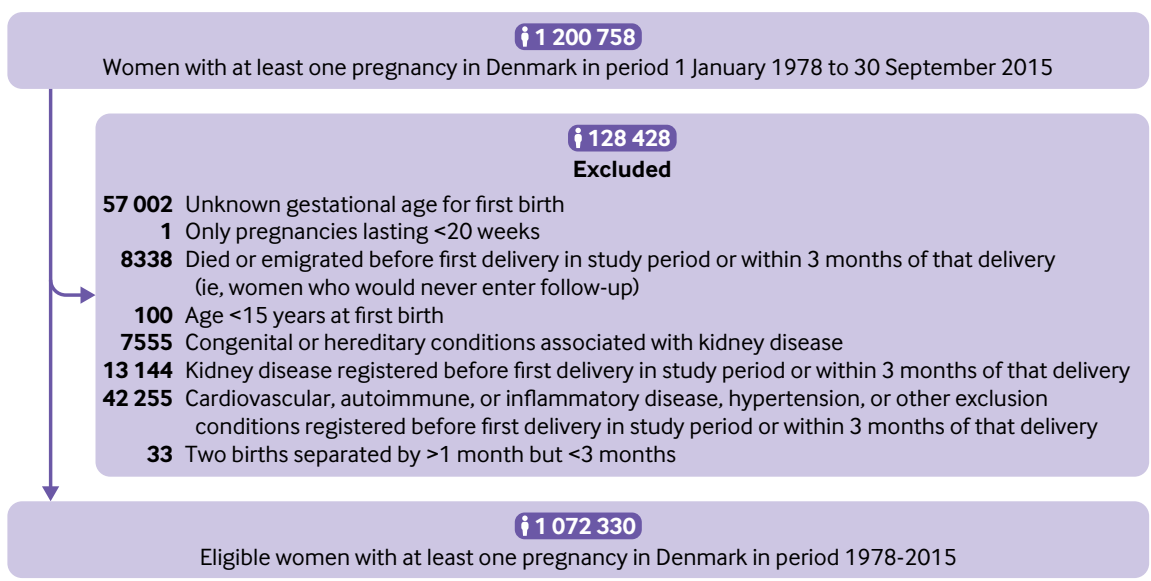

Fig 1 | Flowchart illustrating construction of study cohort 
with gestational hypertension in the period from one month before delivery to seven days postpartum without also receiving a diagnosis of pre-eclampsia. As we did for pre-eclampsia, we treated history of gestational hypertension as a time dependent variable, such that a woman could contribute with both affected and unaffected pregnancies, but once she had experienced gestational hypertension she contributed all remaining person time to the affected group, regardless of any later normotensive pregnancies.

Preterm delivery has been associated with later risk of kidney biopsy, ${ }^{23}$ and it was associated with increased risks of kidney disease in our data. Therefore, we stratified all our main analyses by gestational age at delivery ( $<34$ weeks, 34-36 weeks, $\geq 37$ weeks), so that women with a history of pre-eclampsia who delivered preterm would be compared with women with no history of pre-eclampsia who also delivered preterm. (See supplementary figure A for details of the construction of the time dependent variable used in the stratified analyses that reflected both history of preeclampsia and gestational age at delivery). We used an interaction test to evaluate differences in hazard ratios by gestational age at delivery.

To explore the importance of time since the latest pregnancy, we did additional analyses to estimate separate hazard ratios for time since latest pregnancy less than five years and five years or longer. Owing to power considerations, these analyses could not be stratified on gestational age at delivery. We also explored the effect of adjusting for additional covariates that might be either confounders or mediators of the associations of interest. These covariates included autoimmune and inflammatory conditions, cardiovascular disease, diabetes, and hypertension, all treated as time dependent variables (see supplementary methods for definitions of each group). Therefore, if a woman did not have, for example, diabetes at the start of follow-up but was later diagnosed as having diabetes, she contributed person time as non-diabetic from the start of follow-up until her diagnosis of diabetes; thereafter, she contributed all remaining person time until the end of follow-up as diabetic.

We evaluated potential violations of the proportional hazards assumption by plotting martingale residuals against attained age. ${ }^{24}$ We used SAS statistical software, version 9.4, for all analyses.

\section{Patient and public involvement}

No patients were involved in setting the research question or the outcome measures, nor were they involved in the design or implementation of the study. No patients were asked to advise on interpretation or writing up of results. Direct dissemination of the study results to study participants is not possible given the size of the cohort and length of the study period.

\section{Results}

The study cohort consisted of 1072330 women with 2046984 pregnancies ending in live birth or stillbirth during the study period. The women were followed for 19994470 person years (average 18.6 years per woman). During follow-up, 14816 women developed kidney disease (7320 acute renal disorders (3.6/100000 person years), 3901 chronic renal disorders (1.9/100000 person years), 1470 other specified renal disorders (0.7/100000 person years), and 2125 unspecified renal disorders (1.1/100000 person years)). Of these women, 1062 (7.2\%) had a history of pre-eclampsia. Only 55503 women (5.2\%) were lost to follow-up; most of these women either moved abroad $(n=26835)$ or died $(n=28161)$. Table 1 shows maternal age and parity at first pregnancy in the study period, by pre-eclampsia status and timing of delivery.

Our results showed modest associations between pre-eclampsia later in pregnancy (late preterm and term) and later acute renal disorders, but no statistical support for an association with early preterm preeclampsia (table 2). However, few instances of acute renal disorders occurred among women delivering preterm, particularly among women who also had pre-eclampsia, resulting in wide confidence intervals around the estimates for early preterm pre-eclampsia (table 2).

By contrast, women with a history of preeclampsia had at least twice the risk of later chronic renal disorders overall, compared with women who delivered at the same gestational age but never had pre-eclampsia (table 3). Magnitudes of association were larger for earlier gestational age at delivery $(\mathrm{P}<0.001)$, with the risk of any chronic renal disorder almost quadrupling for women with a history of early preterm pre-eclampsia. We observed a similar pattern of association for three of the four subtypes of chronic renal disorder, although only the pattern for glomerular and proteinuric disease was statistically significant $(\mathrm{P}<0.001)$ (table 3$)$. Few women were diagnosed as having chronic tubulointerstitial nephritis during follow-up, making the drawing of conclusions about this type of chronic renal disorder difficult. Patterns of association for other and unspecified kidney disease were similar to those observed for the chronic renal disorders, whereas the hazard ratios for unspecified tubulointerstitial nephritis more closely resembled the estimates for acute renal disorders (supplementary table A).

When we examined the effect of time since latest pregnancy on our results, pre-eclampsia was especially strongly associated with the risk of chronic kidney disease and glomerular and proteinuric disease within five years of the latest pregnancy (hazard ratio 6.11 (95\% confidence interval 3.84 to 9.72) and 4.77 (3.88 to 5.86), respectively) (fig 2). Thereafter, the strength of the associations decreased (chronic kidney disease, $\mathrm{P}$ for difference $<0.001$; glomerular and proteinuric disease, $\mathrm{P}$ for difference<0.001). However, five years or more after the latest pregnancy, women with a history of pre-eclampsia still had risks of chronic kidney disease and glomerular and proteinuric disease that were $100 \%$ and 50\% higher than the risks observed 


\begin{tabular}{|c|c|c|c|c|c|c|}
\hline \multirow[b]{2}{*}{ Age/parity } & \multicolumn{3}{|c|}{ Pre-eclamptic pregnancy } & \multicolumn{3}{|c|}{ Normotensive pregnancy } \\
\hline & Early preterm delivery & Late preterm delivery & Term delivery & Early preterm delivery & Late preterm delivery & Term delivery \\
\hline Total & $2651(0.2)$ & $4450(0.4)$ & $33524(3.1)$ & $17790(1.7)$ & 41705 (3.9) & $972210(90.7)$ \\
\hline \multicolumn{7}{|c|}{ Maternal age (years): } \\
\hline$<20$ & $89(3.4)$ & $150(3.4)$ & $1257(3.8)$ & $817(4.6)$ & $1735(4.2)$ & $35021(3.6)$ \\
\hline $20-24$ & $594(22.4)$ & $962(21.6)$ & $8749(26.1)$ & $3912(22.0)$ & $9636(23.1)$ & $240541(24.7)$ \\
\hline $25-29$ & $979(36.9$ & $1728(38.8)$ & $13118(39.1)$ & $6339(35.6)$ & $16256(39.0)$ & $394240(40.6)$ \\
\hline $30-34$ & $677(25.5)$ & $1088(24.5)$ & $7176(21.4)$ & $4515(25.4)$ & $9827(23.6)$ & $221316(22.8)$ \\
\hline$\geq 35$ & $312(11.8)$ & $522(11.7)$ & $3224(9.6)$ & $2208(12.4)$ & $4251(10.2)$ & $81092(8.3)$ \\
\hline \multicolumn{7}{|l|}{ Parity*: } \\
\hline 1 & $258897.6)$ & $4313(96.9)$ & $31581(94.2)$ & $16618(93.4)$ & $38995(93.5)$ & $887714(91.3)$ \\
\hline 2 & $59(2.2)$ & $118(2.7)$ & $1631(4.9)$ & $965(5.4)$ & $2249(5.4)$ & $71172(7.3)$ \\
\hline$\geq 3$ & $4(0.2)$ & $19(0.4)$ & $312(0.9)$ & $207(1.2)$ & $461(1.1)$ & $13324(1.4)$ \\
\hline
\end{tabular}

in women with no history of pre-eclampsia (hazard ratio 2.06 (1.69 to 2.50) and 1.50 (1.19 to 1.88), respectively). By contrast, hazard ratios for the acute renal disorders and the other subtypes of chronic renal disorder seemed to be more stable over time (fig 2 and supplementary table B).

Adjustment for diabetes and autoimmune/ inflammatory disease diagnosed during follow-up had little effect on our estimates (table 4). On the other hand, adjustment for post-pregnancy cardiovascular disease and hypertension decreased the strength of the observed associations by $20-30 \%$ (table 4).

\section{Discussion}

Our study found strong, statistically significant associations between pre-eclampsia and later chronic kidney disease, hypertensive kidney disease, and glomerular and proteinuric disease, with stronger associations for earlier onset of pre-eclampsia. The associations between pre-eclampsia and the risks of glomerular and proteinuric disease and chronic kidney disease were particularly strong in the five years after a pregnancy, whereas time since pregnancy did not seem to modify the associations with other types of kidney disease. Adjustment for hypertension and cardiovascular disease diagnosed during the followup period diminished, but could not eliminate, the observed associations with chronic renal disorders, suggesting that the observed associations were only partially mediated by these factors. Conversely, adjustment for diabetes and autoimmune and inflammatory conditions had very little effect on the magnitude of the estimates.

\section{Strengths and potential limitations of study}

Our use of a cohort including all women nationwide who were pregnant during a 38 year period minimised the possibility of selection bias and provided us with the power to examine the effect of timing of onset of preeclampsia, time since pregnancy, and specific kidney disease subtypes. Furthermore, the size of our cohort allowed us to do analyses stratified by gestational age at delivery and to determine that the apparent association between pre-eclampsia and kidney disease was not simply attributable to the preterm delivery often associated with pre-eclampsia but that pre-eclampsia had an added effect. Use of diagnoses recorded in national registers (with mandatory reporting) eliminated the possibility of recall bias and helped to standardise outcome definitions. By applying Cox regression, we were able to accommodate variable follow-up and time dependent covariates that have challenged previous studies.

Although the sensitivity of pre-eclampsia diagnoses recorded in the National Patient Register has been found to be only moderate (69\%), their specificity is very high (>99\%). ${ }^{19}$ Consequently, although a proportion of affected women may not have had their pre-eclampsia registered, somewhat diluting our results, the risk of bias due to unaffected women being classified as having pre-eclampsia was likely negligible. As might be expected in a study spanning decades, definitions

\begin{tabular}{|c|c|c|c|c|c|c|c|c|}
\hline \multirow[b]{2}{*}{ Timing of delivery } & \multirow[b]{2}{*}{ History of PE } & \multirow[b]{2}{*}{ Person years $\left(\times 10^{3}\right)$} & \multicolumn{2}{|c|}{ Any acute renal disorder* } & \multicolumn{2}{|r|}{ Acute kidney failure } & \multicolumn{2}{|c|}{ Acute tubulointerstitial nephritis } \\
\hline & & & No & Hazard ratiot $(95 \% \mathrm{Cl})$ & No & Hazard ratiot $(95 \% \mathrm{Cl})$ & No & Hazard ratiot $(95 \% \mathrm{Cl})$ \\
\hline \multirow[t]{2}{*}{ Early preterm } & Yes & 54.0 & 29 & $1.06(0.72$ to 1.56$)$ & $\neq$ & 0.49 (0.12 to 2.02$)$ & $\neq$ & 1.17 (0.78 to 1.75$)$ \\
\hline & No & 449.1 & 215 & 1 (reference) & 36 & 1 (reference) & 179 & 1 (ref) \\
\hline \multirow[t]{2}{*}{ Late preterm } & Yes & 90.7 & 54 & $1.33(1.00$ to 1.76$)$ & 5 & 0.72 (0.29 to 1.78$)$ & 49 & 1.46 (1.08 to 1.96$)$ \\
\hline & No & 1027.7 & 449 & 1 (reference) & 77 & 1 (reference) & 372 & 1 (ref) \\
\hline \multirow[t]{2}{*}{ Term } & Yes & 760.4 & 313 & $1.12(1.00$ to 1.28$)$ & 59 & 1.57 (1.21 to 2.05$)$ & 254 & $1.05(0.93$ to 1.19$)$ \\
\hline & No & 17612.6 & 6260 & 1 (reference) & 814 & 1 (reference) & 5446 & 1 (ref) \\
\hline $\mathrm{P}$ value for homogeneity & - & - & - & 0.51 & - & 0.09 & - & 0.03 \\
\hline
\end{tabular}




\begin{tabular}{|c|c|c|c|c|c|c|c|c|c|c|c|c|}
\hline \multirow{2}{*}{$\begin{array}{l}\text { Timing of } \\
\text { delivery }\end{array}$} & \multirow{2}{*}{$\begin{array}{l}\text { History } \\
\text { of PE }\end{array}$} & \multirow{2}{*}{$\begin{array}{l}\text { Person years } \\
\left(\times 10^{3}\right)\end{array}$} & \multicolumn{2}{|c|}{$\begin{array}{l}\text { Any chronic renal } \\
\text { disorder }\end{array}$} & \multicolumn{2}{|c|}{$\begin{array}{l}\text { Chronic kidney } \\
\text { disease }\end{array}$} & \multicolumn{2}{|c|}{$\begin{array}{l}\text { Hypertensive kidney } \\
\text { disease }\end{array}$} & \multicolumn{2}{|c|}{$\begin{array}{l}\text { Chronic tubulointerstitial } \\
\text { nephritis }\end{array}$} & \multicolumn{2}{|c|}{$\begin{array}{l}\text { Glomerular and } \\
\text { proteinuric diseases }\end{array}$} \\
\hline & & & No & $\mathrm{HR}^{*}(95 \% \mathrm{CI})$ & No & $\mathrm{HR}^{*}(95 \% \mathrm{Cl})$ & No & $\mathrm{HR}^{*}(95 \% \mathrm{Cl})$ & No & $\mathrm{HR}^{*}(95 \% \mathrm{Cl})$ & No & $\mathrm{HR}^{*}(95 \% \mathrm{Cl})$ \\
\hline \multirow[t]{2}{*}{ Early preterm } & Yes & 54.0 & 62 & $\begin{array}{l}3.93 \\
\text { (2.90 to } 5.33)\end{array}$ & 18 & $\begin{array}{l}2.90 \\
(1.70 \text { to } 4.96)\end{array}$ & 9 & $\begin{array}{l}4.19 \\
\text { (1.86 to } 9.42)\end{array}$ & 5 & $\begin{array}{l}2.91 \\
\text { (1.04 to } 8.11)\end{array}$ & 30 & $\begin{array}{l}5.34 \\
\text { (3.36 to } 8.46)\end{array}$ \\
\hline & No & 449.1 & 130 & 1 (reference) & 53 & 1 (reference) & 17 & 1 (reference) & 14 & 1 (reference) & 46 & 1 (reference) \\
\hline \multirow[t]{2}{*}{ Late preterm } & Yes & 90.7 & 63 & $\begin{array}{l}2.81 \\
(2.13 \text { to } 3.71)\end{array}$ & 17 & $\begin{array}{l}2.24 \\
(1.33 \text { to } 3.78)\end{array}$ & $t$ & $\begin{array}{l}3.69 \\
(1.91 \text { to } 7.13)\end{array}$ & * & $\begin{array}{l}0.93 \\
(0.22 \text { to } 3.95) \\
\end{array}$ & 32 & $\begin{array}{l}3.40 \\
(2.28 \text { to } 5.06)\end{array}$ \\
\hline & No & 1027.7 & 247 & 1 (reference) & 85 & 1 (reference) & 35 & 1 (reference) & 23 & 1 (reference) & 104 & 1 (reference) \\
\hline \multirow[t]{2}{*}{ Term } & Yes & 760.4 & 317 & $\begin{array}{l}2.27 \\
(2.02 \text { to } 2.55) \\
\end{array}$ & 104 & $\begin{array}{l}2.27 \\
(1.85 \text { to } 2.78) \\
\end{array}$ & 64 & $\begin{array}{l}3.06 \\
(2.34 \text { to } 3.99) \\
\end{array}$ & 15 & $\begin{array}{l}1.48 \\
(0.88 \text { to } 2.51) \\
\end{array}$ & 134 & $\begin{array}{l}2.14 \\
(1.79 \text { to } 2.56) \\
\end{array}$ \\
\hline & No & 17612.6 & 3082 & 1 (reference) & 1008 & 1 (reference) & 432 & 1 (reference) & 234 & 1 (reference) & 1408 & 1 (reference) \\
\hline $\begin{array}{l}\text { P value for } \\
\text { homogeneity }\end{array}$ & & & & $<0.001$ & & 0.69 & & 0.70 & & 0.38 & & $<0.001$ \\
\hline
\end{tabular}

HR=hazard ratio; $\mathrm{PE}=$ pre-eclampsia.

Early preterm delivery: delivery <34 weeks. Late preterm delivery: delivery 34-36 weeks. Term delivery: delivery $\geq 37$ weeks.

${ }^{*}$ All hazard ratios are adjusted for maternal age (underlying time in Cox model), maternal birth year, parity, history of gestational hypertension, and history of shortest gestation.

tUnder current Danish and European data protection laws, exact numbers cannot be given because one cell contains $<5$ women.

of pre-eclampsia have evolved since the beginning of our study period. Of particular relevance to our study, proteinuria (a sign of kidney involvement) has no longer been required for a diagnosis of pre-eclampsia according to internationally accepted guidelines since 2014. ${ }^{125}$ However, our follow-up period ended in 2015, only a year after this change came into effect. Furthermore, our study was based on ICD-8 and ICD-10 codes, both of which required proteinuria for a diagnosis of pre-eclampsia (see supplementary methods for definitions). Proteinuria was therefore a fairly universal feature of pre-eclampsia throughout our study period; other changes to diagnostic criteria were unlikely to have produced sufficient heterogeneity in our exposure to have a meaningful effect on the observed associations.

Although the validity of diagnoses of moderate and severe renal disease in the National Patient Register is excellent, ${ }^{21}$ the sensitivity of the registers for individual diagnoses may not always be high, particularly for milder degrees of renal impairment, which are known to be underreported in connection with other comorbidities such as diabetes. ${ }^{26}$ However, the specificity of registered diagnoses of renal disease is likely to be high, particularly for aetiological diagnoses such as glomerular disease, which are not typically assigned without confirmation by biopsy, and for chronic kidney disease, which by definition requires specialist intervention. Any misclassification of outcome is likely to have been non-differential, as postpartum kidney function in women who previously had pre-eclampsia has not been much studied and registration of kidney disorders would not be expected to depend on exposure status. Consequently, underregistration of kidney disease outcomes is unlikely to have biased our results substantially.

The acute renal dysfunction diagnoses included in ICD-8 and ICD-10 changed very little over the study

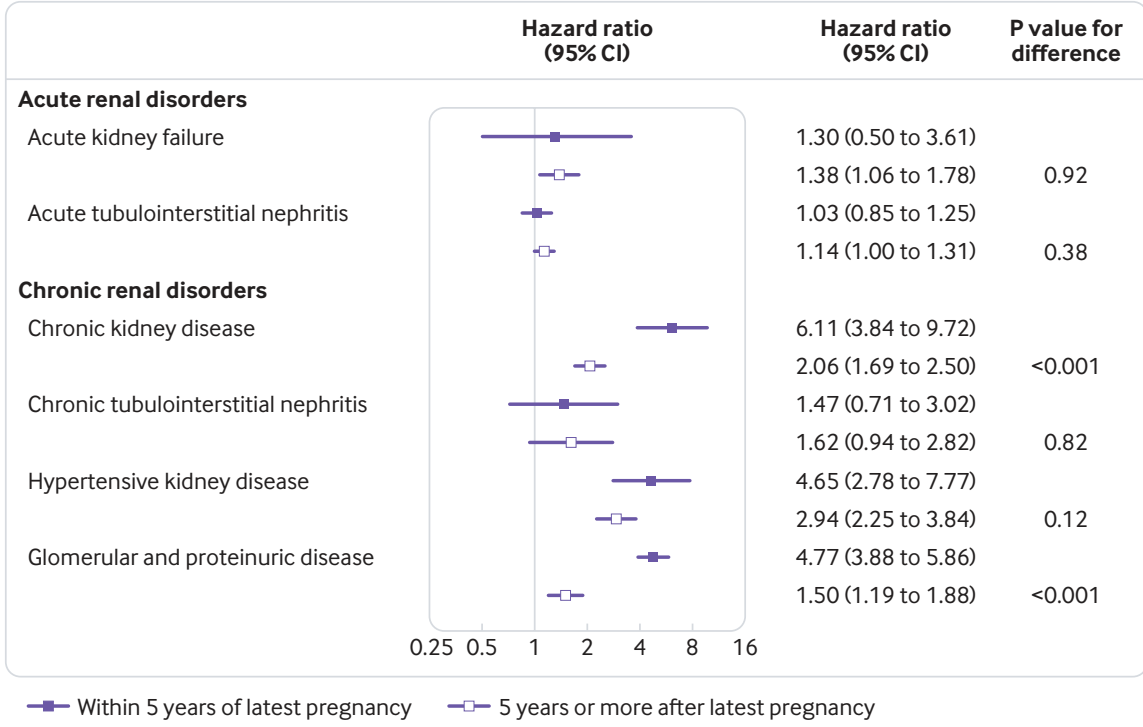

Fig 2 | Hazard ratios for acute and chronic renal disorder groups by time since latest pregnancy, Denmark, 1978-2015. For these analyses, all women with pre-eclampsia were grouped together regardless of gestational age at delivery. Hazard ratios with solid squares compare risks of kidney disease in women with and without history of pre-eclampsia within 5 years of latest pregnancy; estimates with open squares compare risks $\geq 5$ years after latest pregnancy 


\begin{tabular}{|c|c|c|c|c|c|}
\hline \multirow[b]{2}{*}{ Timing of delivery } & \multirow[b]{2}{*}{ No additional adjustment } & \multicolumn{4}{|c|}{ Additionally adjusted for: } \\
\hline & & Autoimmune disease & Cardiovascular disease & Diabetes & Hypertensiont \\
\hline Early preterm & $3.93(2.90$ to 5.33$)$ & $3.88(2.86$ to 5.26$)$ & 2.89 (2.13 to 3.92) & $3.77(2.78$ to 5.11$)$ & $2.84(2.02$ to 4.01$)$ \\
\hline Late preterm & $2.81(2.13$ to 3.71$)$ & $2.74(2.07$ to 3.61$)$ & 2.28 (1.73 to 3.02$)$ & 2.69 (2.04 to 3.56$)$ & $1.83(1.32$ to 2.55$)$ \\
\hline Term & 2.27 (2.02 to 2.55$)$ & 2.23 (1.98 to 2.50$)$ & 1.88 (1.67 to 2.12$)$ & 2.11 (1.87 to 2.37 ) & $1.72(1.51$ to 1.96$)$ \\
\hline
\end{tabular}

period. However, as with pre-eclampsia, kidney disease paradigms have evolved. In particular, the concept of acute kidney injury has emerged in the past 10-15 years, and KDIGO published guidelines for staging acute kidney injury in 2012. ${ }^{27}$ Acute kidney injury and its staging are not yet implemented in the ICD system; of particular note, the early stages of acute kidney injury have no direct equivalent in ICD-10 (the version currently used in Denmark). Consequently, our use of the ICD system will have resulted in an overall underestimation of the occurrence of acute renal dysfunction as defined by the KDIGO system. This underestimation will have been most pronounced for early stages of acute kidney injury, about which little can be inferred from our findings. By contrast, any underestimation of the rates of severe, clinically important acute renal dysfunction is likely to have been negligible, as these conditions are diagnosed and treated in the hospital setting and captured by ICD codes.

Changing definitions and diagnostics for chronic kidney disease have mostly affected our ability to subclassify the condition. Whereas chronic kidney disease was previously classified clinically as mild, moderate, or severe, it is now staged from 1 to $5 .{ }^{6}$ However, the overall definition of chronic kidney disease covered approximately the same group of patients throughout the study period, so changes to definitions and diagnostic criteria are not likely to have had a large effect on our findings.

Pre-eclampsia is strongly associated with later cardiovasculardisease, hypertension, and diabetes, ${ }^{28-30}$ all of which are known risk factors for chronic kidney disease and could therefore be suspected of mediating the observed associations. However, our results indicated that comorbid conditions, cardiovascular disease and hypertension in particular, could only partially explain the observed associations, suggesting that at least part of the link between pre-eclampsia and chronic kidney disease might arise from common predisposing factors such as increased susceptibility to vascular endothelial dysfunction.

Obesity is associated with both pre-eclampsia and chronic kidney disease, but whether obesity might be an independent confounder of the observed associations after adjustment for cardiovascular disease and diabetes (with which it is strongly associated) is unclear. Of the studies that previously examined the association between pre-eclampsia and chronic kidney disease or end stage renal disease, three did not consider obesity important enough to adjust for it. ${ }^{11-13}$ Ayansina and colleagues observed a decrease in magnitude of the association when they adjusted for body mass index, socioeconomic status, and smoking, but they did not also adjust for cardiovascular disease, diabetes, and hypertension. ${ }^{16}$ We lacked information on pre-pregnancy body mass index for most of our cohort, and information on body mass index in non-pregnant individuals is not registered; therefore, we were unable to adjust for the potential influence of obesity. We also cannot rule out the possibility of residual confounding by other unmeasured covariates. Finally, we note that our cohort was comprised predominantly of white European women, which may limit the generalisability of our findings to other populations with different covariate distributions.

\section{Comparison with other studies}

Previous studies of overt renal disease after hypertensive disorders of pregnancy have largely focused on chronic kidney disease, particularly end stage renal disease. Vikse and colleagues found that in women with two pregnancies, a history of preeclampsia increased the risk of end stage renal disease between threefold and sixfold, depending on whether a woman had pre-eclampsia in the first pregnancy, the second pregnancy, or both pregnancies. ${ }^{11}$ Wang and colleagues showed that women with a history of hypertensive disorders of pregnancy had nine times the risk of chronic kidney disease and 12 times the risk of end stage renal disease, compared with women who had no such history; consistent with our results, adjustment for hypertension and other comorbidities attenuated the estimates, although hypertensive disorders of pregnancy were still associated with twofold to threefold increases in risk of later kidney disease. ${ }^{12}$ Similarly, $\mathrm{Wu}$ and colleagues found that a history of hypertensive disorders of pregnancy was associated with a 10-fold increase in the risk of end stage renal disease. ${ }^{13}$ Finally, Ayansina and colleagues found that pre-eclampsia was associated with a twofold increase in risk of later kidney insufficiency that the KDIGO guidelines would classify as chronic kidney disease. ${ }^{16}$ Our results add nuance to this picture, showing that the risk of chronic renal disorders was substantially elevated in women with previous 
pre-eclampsia regardless of the timing of onset of pre-eclampsia, but that women who had preterm pre-eclampsia were especially at risk. Furthermore, we showed that the risks of post-pregnancy chronic kidney disease and glomerular and proteinuric disease were greatest in the five years after a pregnancy, rather than decades later.

Our findings for the glomerular and proteinuric diseases are consistent with the results of other studies suggesting that microalbuminuria and possibly reduced glomerular filtration rate are more prevalent among women with previous pre-eclampsia than among women who had healthy pregnancies. $^{7} 10$ Interestingly, we found that women with a history of pre-eclampsia were at greatest risk of glomerular and proteinuric disease within five years of pregnancy.

Although acute kidney failure is a known complication of pre-eclampsia, particularly in developing countries, ${ }^{31}$ little is known about the relation between pre-eclampsia and incident acute renal disorders postpartum. We found evidence of only modest associations between pre-eclampsia and later acute renal disorders and then only for pre-eclampsia with onset later in pregnancy (later preterm and term pre-eclampsia). However, very few women with a history of preterm pre-eclampsia developed acute renal disorders during the follow-up period, preventing us from drawing firm conclusions about whether an association exists between preterm pre-eclampsia and acute kidney disease and, if so, how strong such an association might be. Associations between preeclampsia and acute renal disorders may reflect shared susceptibility to both pathologies conferred by common risk factors. Alternatively, undetected prepregnancy acute kidney dysfunction in some women may have contributed to the observed associations. We also cannot exclude the possibility that the associations we observed between pre-eclampsia of later onset and acute renal disorders might have resulted from detection bias, as women with pre-eclampsia are intensively monitored after delivery to ensure that their creatinine concentrations return to normal. However, our decision to ignore diagnoses of kidney disease registered within three months of delivery and to stop follow-up for these women should have minimised the effect of any such bias.

\section{Conclusions and policy implications}

Our study results suggest that at least a subset of women with previous pre-eclampsia need clinical monitoring for kidney disease in the years immediately after pregnancy. Early detection of chronic renal disorders would allow for early intervention to delay disease progression $^{32} 33$; ideally, detection of the antecedents of these disorders would permit implementation of measures to prevent the onset of chronic kidney disease entirely. However, more research is needed to determine which women are at greatest risk of kidney disease after pre-eclampsia, what mechanisms underlie the association, and therefore what follow- up and interventions (and in what timeframe postpregnancy) would be most appropriate and effective.

Contributors: JHK conceived and designed the study; defined exclusion criteria and exposure, outcome, and covariate categories; interpreted the study findings; and drafted the manuscript. SB conceived and designed the study, acquired and analysed the data, interpreted the study findings, and critically reviewed the manuscript. JW designed the study, advised on selection and implementation of analytical methods, interpreted the study findings, and critically reviewed the manuscript. MBD conceived and designed the study, interpreted the study findings, and critically reviewed the manuscript. $\mathrm{HAB}$ conceived and designed the study, supervised and directed the conduct of the study, interpreted the study findings, and critically revised the manuscript. The corresponding author attests that all listed authors meet authorship criteria and that no others meeting the criteria have been omitted. JK, SB, JW, and HAB had full access to all of the data in the study and can take responsibility for the integrity of the data and the accuracy of the data analysis. HAB is the guarantor.

Funding: This work was partially supported by the Danish Council for Independent Research (DFF-4092-00213). The Danish Council for Independent Research had no role in the design and conduct of the study; collection, management, analysis, and interpretation of the data; preparation, review, or approval of the manuscript; or the decision to submit the manuscript for publication. The researchers acted independently from the study sponsor in all aspects of this study.

Competing interests: All authors have completed the ICMJE uniform disclosure form at www.icmje.org/coi_disclosure.pdf (available on request from the corresponding author) and declare: SB was supported by a grant from the Danish Council for Independent Research; no other support for the submitted work; no financial relationships with any organisations that might have an interest in the submitted work in the previous three years; no other relationships or activities that could appear to have influenced the submitted work.

Ethical approval: Studies based solely on data from the Danish national registers do not need approval from the Danish research bioethics committees, as study participants are never contacted, and consent is not required for the use of register information. The study's use of register data was covered by the approval extended by the Danish Data Protection Agency to all register based studies conducted by Statens Serum Institut (approval No 2015-57-0102).

Data sharing: This study is based on Danish national register data. These data do not belong to the authors but to the Danish Ministry of Health, and the authors are not permitted to share them, except in aggregate (as, for example, in a publication). However, interested parties can obtain the data on which the study was based by submitting a research protocol to the Danish Data Protection Agency (Datatilsynet) and then, once Data Protection Agency permission has been received, applying to the Ministry of Health's Research Service (Forskerservice) at forskerservice@ssi.dk.

Transparency: The manuscript's guarantor (HAB) affirms that the manuscript is an honest, accurate, and transparent account of the study being reported; that no important aspects of the study have been omitted; and that any discrepancies from the study as planned (and, if relevant, registered) have been explained.

This is an Open Access article distributed in accordance with the Creative Commons Attribution Non Commercial (CC BY-NC 4.0) license, which permits others to distribute, remix, adapt, build upon this work non-commercially, and license their derivative works on different terms, provided the original work is properly cited and the use is noncommercial. See: http://creativecommons.org/licenses/by-nc/4.0/.

Brown MA, Magee LA, Kenny LC, et al, International Society for the Study of Hypertension in Pregnancy (ISSHP). The hypertensive disorders of pregnancy: ISSHP classification, diagnosis \& management recommendations for international practice. Pregnancy Hypertens 2018;13:291-310. doi:10.1016/j.preghy.2018.05.004

2 Strevens H, Wide-Swensson D, Hansen A, et al. Glomerular endotheliosis in normal pregnancy and pre-eclampsia. BJOG 2003;110:831-6. doi:10.1111/j.1471-0528.2003.02162.x

3 Henao DE, Saleem MA. Proteinuria in preeclampsia from a podocyte injury perspective. Curr Hypertens Rep 2013;15:600-5. doi:10.1007/s11906-013-0400-1

4 Craici IM, Wagner SJ, Weissgerber TL, Grande JP, Garovic VD. Advances in the pathophysiology of pre-eclampsia and related podocyte injury. Kidney Int 2014;86:275-85. doi:10.1038/ki.2014.17 
5 Furuta I, Zhai T, Ishikawa S, et al. Association between nephrinuria, podocyturia, and proteinuria in women with pre-eclampsia. J Obstet Gynaecol Res 2017;43:34-41. doi:10.1111/jog.13180

6 Kidney Disease, Improving Global Outcomes (KDIGO) CKD Work Group. KDIGO clinical practice guideline for the evaluation and management of chronic kidney disease. Kidney Int Suppl 2013;3:1150.

7 Lopes van Balen VA, Spaan JJ, Cornelis T, Spaanderman MEA. Prevalence of chronic kidney disease after preeclampsia. I Nephrol 2017;30:403-9. doi:10.1007/s40620-016-0342-1

8 Sandvik MK, Hallan S, Svarstad E, Vikse BE. Preeclampsia and prevalence of microalbuminuria 10 years later. Clin J Am Soc Nephrol 2013;8:1126-34. doi:10.2215/CJN.10641012

9 Lopes van Balen VA, Spaan JJ, Cornelis T, Heidema WM, Scholten RR, Spaanderman MEA. Endothelial and kidney function in women with a history of preeclampsia and healthy parous controls: A case control study. Microvasc Res 2018;116:71-6. doi:10.1016/j. mvr.2017.11.001

10 McDonald SD, Han Z, Walsh MW, Gerstein HC, Devereaux PJ. Kidney disease after preeclampsia: a systematic review and meta-analysis. Am J Kidney Dis 2010;55:1026-39. doi:10.1053/j. ajkd.2009.12.036

11 Vikse BE, Irgens LM, Leivestad T, Skjaerven R, Iversen BM. Preeclampsia and the risk of end-stage renal disease. $N$ Engl $)$ Med 2008;359:800-9. doi:10.1056/NEJMoa0706790

12 Wang IK, Muo CH, Chang YC, et al. Association between hypertensive disorders during pregnancy and end-stage renal disease: a population-based study. CMAI 2013;185:207-13. doi:10.1503/ cmaj.120230

13 Wu CC, Chen SH, Ho CH, et al. End-stage renal disease after hypertensive disorders in pregnancy. Am J Obstet Gynecol 2014:210:147. doi:10.1016/i.ajog.2013.09.027

14 Schokker SA, Van Oostwaard MF, Melman EM, et al. Cerebrovascular, cardiovascular and renal hypertensive disease after hypertensive disorders of pregnancy. Pregnancy Hypertens 2015;5:287-93. doi:10.1016/j.preghy.2015.06.002

15 Paauw ND, van der Graaf AM, Bozoglan R, et al. Kidney function afte a hypertensive disorder of pregnancy: a longitudinal study. Am J Kidney Dis 2018;71:619-26. doi:10.1053/j.ajkd.2017.10.014

16 Ayansina D, Black C, Hall SJ, et al. Long term effects of gestational hypertension and pre-eclampsia on kidney function: Record linkage study. Pregnancy Hypertens 2016;6:344-9. doi:10.1016/j. preghy.2016.08.231

17 Knudsen LB, Olsen J. The Danish Medical Birth Registry. Dan Med Bull 1998;45:320-3.

18 Lynge E, Sandegaard IL, Rebolj M. The Danish National Patient Register. Scand J Public Health 2011;39(Suppl):30-3. doi:10.1177/1403494811401482

19 Klemmensen AK, Olsen SF, Osterdal ML, Tabor A. Validity of preeclampsia-related diagnoses recorded in a national hospital registry and in a postpartum interview of the women. $\mathrm{Am}$ / Epidemiol 2007;166:117-24. doi:10.1093/aje/kwm139
20 Helweg-Larsen K. The Danish Register of Causes of Death. Scand I Public Health 2011;39(Suppl):26-9. doi:10.1177/1403494811399958

21 Thygesen SK, Christiansen CF, Christensen S, Lash TL, Sørensen $H T$. The predictive value of ICD-10 diagnostic coding used to assess Charlson comorbidity index conditions in the populationbased Danish National Registry of Patients. BMC Med Res Methodol 2011;11:83. doi:10.1186/1471-2288-11-83

22 Schmidt M, Pedersen L, Sørensen HT The Danish Civil Registration System as a tool in epidemiology. Eur J Epidemiol 2014;29:541-9. doi:10.1007/s10654-014-9930-3

23 Vikse BE, Irgens LM, Bostad L, Iversen BM. Adverse perinatal outcome and later kidney biopsy in the mother. I Am Soc Nephrol 2006;17:837-45. doi:10.1681/ASN.2005050492

24 Lin DY, Wei LJ, Ying Z. Checking the Cox model with cumulative sums of martingale-based residuals. Biometrika 1993;80:557-72. doi:10.1093/biomet/80.3.557

25 Tranquilli AL, Dekker G, Magee L, et al. The classification, diagnosis and management of the hypertensive disorders of pregnancy: A revised statement from the ISSHP. Pregnancy Hypertens 2014;4:97104. doi:10.1016/j.preghy.2014.02.001

26 Middleton RJ, Foley RN, Hegarty J, et al. The unrecognized prevalence of chronic kidney disease in diabetes. Nephrol Dial Transplant 2006;21:88-92. doi:10.1093/ndt/gfi163

27 Kidney Disease, Improving Global Outcomes (KDIGO) AKI Work Group. KDIGO clinical practice guideline for acute kidney injury. Kidney Int Suppl 2012;2:1-138.

28 Brown MC, Best KE, Pearce MS, Waugh J, Robson SC, Bell R. Cardiovascular disease risk in women with pre-eclampsia: systematic review and meta-analysis. Eur J Epidemiol 2013;28:1-19. doi:10.1007/s10654-013-9762-6

29 Behrens I, Basit S, Melbye M, et al. Risk of post-pregnancy hypertension in women with a history of hypertensive disorders of pregnancy: nationwide cohort study. BMJ 2017;358:j3078 doi:10.1136/bmj.j3078

30 Lykke JA, Langhoff-Roos J, Sibai BM, Funai EF, Triche EW, Paidas MJ. Hypertensive pregnancy disorders and subsequent cardiovascular morbidity and type 2 diabetes mellitus in the mother. Hypertension 2009;53:944-51. doi:10.1161/ HYPERTENSIONAHA.109.130765

31 Prakash J, Niwas SS, Parekh A, et al. Acute kidney injury in late pregnancy in developing countries. Ren Fail 2010;32:309-13. doi:10.3109/08860221003606265

32 Hommel K, Madsen M, Kamper AL. The importance of early referral for the treatment of chronic kidney disease: a Danish nationwide cohort study. BMC Nephrol 2012:13:108 doi:10.1186/1471-2369-13-108

33 Lonnemann G, Duttlinger J, Hohmann D, Hickstein L, Reichel H. Timely referral to outpatient nephrology care slows progression and reduces treatment costs of chronic kidney diseases. Kidney Int Rep 2016:2:142-51. doi:10.1016/j.ekir.2016.09.062

\section{Supplementary materials}

